\title{
Antimicrobial profile of inducible clindamycin resistant strains of staphylococcus species
}

\author{
Sunil Hatkar ${ }^{1, *}$, Sucheta Lakhani ${ }^{2}$, Santosh Kotgire ${ }^{3}$ \\ ${ }^{\mathbf{1}}$ Assistant Professor, ${ }^{2}$ Professor, ${ }^{\mathbf{3}}$ Associate Professor, Dept. of Microbiology, ${ }^{1}$ SMBT Medical College, Nashik, Maharashtra, \\ ${ }^{2}$ SBKS Medical College \& Sumandeep University, Vadodara, Gujarat, ${ }^{3}$ IIMSR Medical College, Warudi, Jalna, Maharashtra, \\ India
}

*Corresponding Author:

Email: sunilhatkar25@gmail.com

\begin{abstract}
Introduction: The appearance of continuous resistant to multiple drugs among Staphylococci is a global burden due to its ability to cause severe infections. The selective use of drugs is necessary to overwhelm the situation. Taking in account present study was carried out to rule out true susceptibility of clindamycin towards staphylococcus species and its antimicrobial profile for judicial use of the drugs.

Material and Methods: All the clinical samples received in the Department of microbiology were screened for Staphylococci as per standard guidelines which were further subjected to Antimicrobial susceptibility testing and D-test to detect MLSb phenotypes.

Results: A total of 421 Staphylococcus species, 359(85.3\%) were Staphylococcus aureus and 62(14.8\%) were Coagulase negative Staphylococci; among them 42(10\%) were Staphylococcus epidermidis\& 20(4.8\%) were Staphylococcus saprophyticus. D-test for S.aureus shows that 173(48.2\%) inducible Clindamycin resistant, $113(31.5 \%)$ strains were constitutive MLSb phenotypes and 58(16.2\%) strains shown to have MSb phenotypes. Among CoNS; among Staphylococcus epidermidis and S.saprophyticus $9.5 \%$ \& 5\% were Inducible Clindamycin resistance, $38.1 \%$ \& $85 \%$ were constitutive MLSb phenotypes and $28.6 \% \& 10 \%$ were MSb phenotypes respectively. All the isolates were sensitive to Linezolid, Vancomycin and Ceftaroline.

Conclusion: Inducible clindamycin resistant strains of Staphylococci found to be among half of the strains, indicating that true susceptibility of clindamycin should be rule out on routine basis for proper institution of the therapy.
\end{abstract}

Keywords: Staphylococci, MLSb phenotypes, AST.

\section{Introduction}

A notorious pathogen, which is gram positive cocci arranged in clusters, is making the condition worsen day by day with acquisition of multidrug resistance. Specifically, Staphylococcus aureus have left use very few therapeutic alternatives to treat such infection. ${ }^{1,2,3}$ Staphylococcus aureus causes variety of infection range from minor skin and soft tissue infection to life threatening condition like endocarditis, septicemia, toxic shock syndrome, Osteomyelitisetc. ${ }^{4,5}$

Emergence of methicillin resistant strains of Staphylococcus aureus is one of the major concerns.First resistance to this organism was noted in year of 1930 for sulfonamide, which was tackled by benzyl penicillin in 1941. The continuous uses of penicillin cause selection of resistant strain by the production of beta-lactamases enzyme. Introduction of synthetic penicillin like methicillin and cloxacillinwere seems to be control of, but in the year of 1962, methicillin resistant Staphylococcus species has started to emerge, that have evolved resistance to all penicillin group of drugs, newer synthetic penicillins and cephalosporins. Methicillin resistant strains of Staphylococcus aureus is mediated by the production of low affinity Penicillin binding protein $2 \mathrm{a}$ encoded by mecAgenes. ${ }^{6-8}$

The macrolide-lincosamide-streptogramin B (MLSb) group of antibiotics found to be good therapeutic alternative to treat MRSA, with Clindamycin being preferred agent due to its excellent pharmacokinetic properties. In recent year, resistant to MLSb antibiotics has started to emerge, additionally inducible resistance to Clindamycin under the influence of erm genes lead to therapeutic failure. ${ }^{9,10}$

The MLSb group of drugs interact with 30s ribosomal subunit to inhibit protein synthesis of bacterial genes. The erythromycin (Macrolide) resistant strains of staphylococci enhance the production of methylase enzyme encoded by erm genes which modifies the target site and might predict the resistance to other group of drugs (Clindamycin).The target site modification either expressed inducible or constitutively, and these strains are difficult to detect as they appear as erythromycin resistant and Clindamycin sensitive in-vitro. In such case, in-vivo therapy with Clindamycin, erm genes mutant may be express constitutively resulting in therapeutic failure. Second mechanism of resistance to MLSb group antibiotics is presence of efflux pump encoded by msrA genes which leads to resistance to Macrolide and Streptogramin-B but not to Lincosamide known as MSb phenotypes. The genotypic detection of ermgenes can be done, but it is costly and inconvenient in resource constraint settings, CLSI recommend phenotypic D-test which is simple, reliable, inexpensive, can be perform on routine basis. ${ }^{11,12}$ 
The present study was carried out to detect inducible Clindamycin resistant strains of staphylococcus species by using simple D-test and their antimicrobial susceptibility profile to find out the resistance pattern at our area.

\section{Material and Methods}

Present study was carried out in the Department of microbiology. After the clearance of RAC and IEC, all the clinical samples received from in-patients and outpatients were analysed for the isolation of pathogen. A total of 421 Staphylococcus species were isolated as per standard guidelines. All the isolates were further antimicrobial susceptibility testing by using KirbyBauer disc diffusion method as per CLSI guidelines. Those isolates which were resistant to erythromycin and sensitive to Clindamycin were evaluated to detect MLSb phenotypes by using D-test. ${ }^{13,14}$ Briefly; Erythromycin $(15 \mu \mathrm{g})$ disc was placed at a distance of $15 \mathrm{~mm}$ (edge to edge) from Clindamycin $(2 \mu \mathrm{g})$ disc on a MHA plate previously inoculated with $0.5 \mathrm{McFarland}$ bacterial suspensions, incubated at $37^{\circ} \mathrm{C}$ for 24 hours and result was interpreted as per CLSI guidelines as follows: ${ }^{15}$

1. MSb phenotype: Staphylococcus species exhibiting resistance to Erythromycin (Zone size $\leq$ $13 \mathrm{~mm}$ ) and sensitive to Clindamycin (Zone size $\geq$
$21 \mathrm{~mm}$ ) and givingcircular zone of inhibition around Clindamycin disc.

2. Inducible MLSb phenotype: Staphylococcus species exhibiting resistance to Erythromycin (Zone size $\leq 13 \mathrm{~mm}$ ) and sensitive to Clindamycin (Zone size $\geq 21 \mathrm{~mm}$ ) and giving $\mathrm{D}$ shaped zone of inhibition around Clindamycin disc.

3. Constitutive MLSb phenotype: Staphylococcus species exhibiting resistance to both Erythromycin (Zone size $\leq 13 \mathrm{~mm}$ ) and Clindamycin (Zone size $\leq 14 \mathrm{~mm}$ ) withgiving circular shape of zone of inhibition if any around Clindamycin disc.

\section{Statistical Analysis}

It was done by using IBM SPSS (20 version) software. Frequencies \& percentages were calculated for all the parameters. Non Parametric test was run by selecting one sample, in which automatically compares observed data to hypothesized using the Chi-Square test.

\section{Observation and Result}

A total of 421 Staphylococcus species isolated from various clinical samples, out of which 359 were Staphylococcus aureus and 62 were CoNS. All the isolates were subjected to detection of methicillin resistance and MLSb phenotypes.

Table 1: Methicillin resistant strains of Staphylococcus species

\begin{tabular}{|c|c|c|c|c|c|}
\hline Methicillin resistance & Frequency & Percent & Valid Percent & Cumulative Percent \\
\hline \multirow{3}{*}{ S.aureus } & MRSA & 280 & 78.0 & 78.0 & 78.0 \\
\cline { 2 - 6 } & MSSA & 79 & 22.0 & 22.0 & 100.0 \\
\cline { 2 - 6 } & Total & 359 & 100.0 & 100.0 & \\
\hline \multirow{3}{*}{ CoNS } & MRCNSS & 44 & 71.0 & 71.0 & 71.0 \\
\cline { 2 - 6 } & MSCNSS & 18 & 29.0 & 29.0 & 100.0 \\
\cline { 2 - 6 } & Total & 62 & 100.0 & 100.0 & \\
\hline
\end{tabular}

Table 2: Frequency of MLSb phenotype among Staphylococcus aureus

\begin{tabular}{|l|c|c|c|c|}
\hline \multicolumn{1}{|c|}{ Type of resistance } & Frequency & Percent & $\begin{array}{c}\text { Valid } \\
\text { Percent }\end{array}$ & Cumulative Percent \\
\hline ER-Sensitive & 15 & 4.1 & 4.1 & 4.1 \\
\hline ER-R, CD-S (iMLSb) & 113 & 31.5 & 31.5 & 35.6 \\
\hline ER-R, CD-R (cMLSb) & 173 & 48.2 & 48.2 & 83.8 \\
\hline ER-R, CD-S (MSb) & 58 & 16.2 & 16.2 & 100.0 \\
\hline Total & 359 & 100.0 & 100.0 & \\
\hline $\begin{array}{l}\text { One-sample Chi- } \\
\text { square test }\end{array}$ & $\begin{array}{l}\text { Test statistics: } 156.733 \\
\text { Degree of freedom: } 3 \\
\text { Asymptomatic p-value(2-sided test): } 0.000\end{array}$ \\
\hline
\end{tabular}

Table 3: Distribution of MLSb phenotype among MRSA and MSSA isolates

\begin{tabular}{|l|c|c|c|c|}
\hline \multirow{2}{*}{ Type of resistance } & \multicolumn{2}{|c|}{ MRSA (n=280) } & \multicolumn{2}{c|}{ MSSA (n=79) } \\
\cline { 2 - 5 } & Frequency & Percent & Frequency & Percent \\
\hline ER-sensitive & 0 & 0 & 15 & 19 \\
\hline iMLSb phenotype & 101 & 36.1 & 12 & 15.2 \\
\hline cMLSb phenotype & 162 & 57.9 & 11 & 13.9 \\
\hline MSb phenotype & 17 & 6 & 41 & 51.9 \\
\hline Total & 280 & 100 & 79 & 100 \\
\hline
\end{tabular}


Table 4: Frequency of MLSb phenotypes among CoNS

\begin{tabular}{|c|c|c|c|c|c|}
\hline CoNS & Phenotypes & Frequency & Percent & $\begin{array}{c}\text { Valid } \\
\text { Percent }\end{array}$ & $\begin{array}{c}\text { Cumulative } \\
\text { Percent }\end{array}$ \\
\hline \multirow{5}{*}{ S.epidermidis } & ER-Sensitive & 10 & 23.8 & 23.8 & 23.8 \\
\hline & $\mathrm{cMLSb}$ & 16 & 38.1 & 38.1 & 61.9 \\
\hline & iMLSb & 4 & 9.5 & 9.5 & 71.4 \\
\hline & $\mathrm{MSb}$ & 12 & 28.6 & 28.6 & 100.0 \\
\hline & Total & 42 & 100.0 & 100.0 & \\
\hline \multirow{5}{*}{ S.saprophyticus } & ER-Sensitive & 0 & 0 & 0 & \\
\hline & iMLSb & 1 & 5.0 & 5.0 & 5.0 \\
\hline & $\mathrm{cMLBb}$ & 17 & 85.0 & 85.0 & 90.0 \\
\hline & $\mathrm{MSb}$ & 2 & 10.0 & 10.0 & 100.0 \\
\hline & Total & 20 & 100.0 & 100.0 & \\
\hline \multicolumn{2}{|c|}{ One-sample Chi-square test } & \multicolumn{4}{|c|}{$\begin{array}{l}\text { Test statistics: } 7.143 \\
\text { Degree of freedom: } 3 \\
\text { Asymptomatic p-value(2-sided test): } 0.067\end{array}$} \\
\hline
\end{tabular}

Table 5: Distribution of MLSbphenotype among MRCNSS and MSCNSS

\begin{tabular}{|l|c|c|c|c|}
\hline \multirow{2}{*}{ Type of strains } & \multicolumn{4}{|c|}{ Type of resistance } \\
\cline { 2 - 5 } & iMLSb & cMLSb & MSb & Total \\
\hline MRCNSS $(\mathrm{n}=44)$ & 04 & 31 & 03 & 38 \\
\hline MSCNSS $(\mathrm{n}=18)$ & 02 & 00 & 11 & 13 \\
\hline Total & 06 & 31 & 14 & 51 \\
\hline
\end{tabular}

Table 6: Antibiotic sensitivity pattern of MLSbphenotypes Staphylococcal aureus

\begin{tabular}{|l|c|c|c|c|c|c|}
\hline \multirow{2}{*}{ Antibiotics } & \multicolumn{2}{|c|}{ iMLSb(n=113) } & \multicolumn{2}{c|}{$\mathbf{c M L S b}(\mathrm{n}=173)$} & \multicolumn{2}{c|}{ MSb(n=58) } \\
\cline { 2 - 7 } & $\mathbf{S ( \% )}$ & $\mathbf{R}(\boldsymbol{\%})$ & $\mathbf{S}(\boldsymbol{\%})$ & $\mathbf{R}(\boldsymbol{\%})$ & $\mathbf{S}(\boldsymbol{\%})$ & $\mathbf{R}(\boldsymbol{\%})$ \\
\hline Erythromycin & 00 & $113(100)$ & 00 & $173(100)$ & 00 & $58(100)$ \\
\hline Clindamycin & $113(100)$ & 00 & 00 & $173(100)$ & $58(100)$ & 00 \\
\hline Cefoxitin & $12(10.6)$ & $101(89.4)$ & $11(6.4)$ & $162(93.6)$ & $41(70.7)$ & $17(29.3)$ \\
\hline Penicillin & 00 & $113(100)$ & 00 & $173(100)$ & 00 & $58(100)$ \\
\hline Trimetho-sulfa & $05(4.5)$ & $108(95.5)$ & $67(38.8)$ & $106(61.2)$ & $39(67.2)$ & $19(32.8)$ \\
\hline Ceftaroline & $113(100)$ & 00 & $173(100)$ & 00 & $58(100)$ & 00 \\
\hline Linezolid & $113(100)$ & 00 & $173(100)$ & 00 & $58(100)$ & 00 \\
\hline Tetracycline & $16(14.2)$ & $97(85.8)$ & 00 & $173(100)$ & $41(70.7)$ & $17(29.3)$ \\
\hline Vancomycin & $113(100)$ & 00 & $173(100)$ & 00 & $58(100)$ & 00 \\
\hline Rifampin & $113(100)$ & 00 & $173(100)$ & 00 & $55(94.8)$ & $03(5.2)$ \\
\hline Chloramphenicol & $16(14.2)$ & $97(85.8)$ & $27(15.6)$ & $146(84.4)$ & $06(10.4)$ & $52(89.6)$ \\
\hline Ofloxacin & 00 & $113(100)$ & $16(9.3)$ & $157(90.7)$ & $45(77.6)$ & $13(22.4)$ \\
\hline Gentamycin & $15(13.3)$ & $98(86.7)$ & $16(9.3)$ & $157(90.7)$ & $38(65.5)$ & $20(34.5)$ \\
\hline
\end{tabular}

Table 7: Antibiotic sensitivity pattern of erythromycin resistant CONS

\begin{tabular}{|l|c|c|c|c|c|c|c|c|}
\hline Antimicrobial agents & \multicolumn{4}{|c|}{ S.epidermidis $=\mathbf{4 2}$} & \multicolumn{4}{c|}{ S.saprophyticus $=\mathbf{2 0}$} \\
\hline & S & \% & $\mathbf{R}$ & $\mathbf{\%}$ & $\mathbf{S}$ & $\mathbf{\%}$ & $\mathbf{R}$ & $\mathbf{\%}$ \\
\hline Erythromycin & 10 & 23.8 & 32 & 76.2 & 01 & 5 & 19 & 95 \\
\hline Clindamycin & 26 & 61.9 & 16 & 38.1 & 05 & 25 & 15 & 75 \\
\hline Cefoxitin & 18 & 42.8 & 24 & 57.2 & 00 & 00 & 20 & 100 \\
\hline Penicillin & 00 & 00 & 42 & 100 & 00 & 00 & 20 & 100 \\
\hline Trimethoprim-sulfa & 07 & 16.6 & 35 & 83.4 & 08 & 40 & 12 & 60 \\
\hline Ceftaroline & 42 & 100 & 00 & 00 & 20 & 100 & 00 & 00 \\
\hline Linezolid & 42 & 100 & 00 & 00 & 20 & 100 & 00 & 00 \\
\hline Tetracycline & 22 & 52.4 & 20 & 47.6 & 04 & 20 & 16 & 80 \\
\hline Vancomycin & 42 & 100 & 00 & 00 & 20 & 100 & 00 & 00 \\
\hline Rifampin & 40 & 95.2 & 02 & 4.8 & 19 & 95 & 01 & 5 \\
\hline Chloramphenicol & 21 & 50 & 21 & 50 & 16 & 80 & 04 & 20 \\
\hline Ofloxacin & 06 & 14.3 & 36 & 85.7 & 16 & 80 & 04 & 20 \\
\hline Gentamycin & 19 & 45.2 & 23 & 54.8 & 13 & 65 & 07 & 35 \\
\hline
\end{tabular}




\section{Discussion}

A total of 421 Staphylococcus species were isolated from different clinical specimens. Out of which, 359(85.3\%) were Staphylococcus aureus and 62(14.8\%) were Coagulase negative Staphylococci; among them $42(10 \%)$ were Staphylococcus epidermidis\& 20(4.8\%) were Staphylococcus saprophyticus.

Inducible Clindamycin resistant strains were found out among erythromycin resistant strains of Staphylococcus species by using D-test. The test was performed by placing erythromycin and Clindamycin disc at $15 \mathrm{~mm}$ distance from edge to edge. The Interdisc distance of $15 \mathrm{~mm}$ has been found to satisfactory by Ajanta G.S. etal ${ }^{16}$ and Fiebelkorn K.R et al. ${ }^{17}$ In our study same protocol of keeping inter-disc distance of 15 mm was followed.

In present study, out of 359 Staphylococcus aureus isolates, 344(95.8\%) were erythromycin resistant, these were further subjected to D-test. The D-test revealed MLSb phenotypes among Staphylococcus aureus, $113(31.5 \%)$ strains were D-test positive indicating inducible Clindamycin resistant strains of Staphylococcus aureus (iMLSb phenotype), 173(48.2\%) strains were constitutive MLSb phenotypes and 58(16.2\%) strains shown to have MSb phenotypes. Similar studies were conducted by Sunil Hatkaret.al ${ }^{10}$ (iMLSb phenotypes 26.13\%, cMLSb phenotypes 58.52\%, and MSbphenotypes15.34\%), VeenaManjunathetal $^{18}$ (iMLSb phenotypes 33.33\%, cMLSb phenotypes $18.75 \%$, and MSb phenotypes 47.9\%) which is in concordance with present study.

Prevalence of MLSb phenotypes among MRSA and MSSA were analysed and it was observed that inducible and constitutive Clindamycin resistant strains of Staphylococcus aureus were higher amongst MRSA isolates (36.1\% \& $57.9 \%$ respectively) as compared to MSSA isolates $(15.2 \%$ \& $13.9 \%$ respectively). In a study of VeenaManjunathetal, ${ }^{18}$ percentages of inducible Clindamycin resistance were higher among MRSA as compared to MSSA (57.63\% and $16.22 \%$ respectively).

The coagulase negative staphylococci also screened for Inducible Clindamycin resistant strains. Among Staphylococcus epidermidis and S.saprophyticus $9.5 \% \quad \& \quad 5 \%$ were Inducible Clindamycin resistance, $38.1 \%$ \& $85 \%$ were constitutive MLSb phenotypes and $28.6 \% \& 10 \%$ were MSb phenotypes.

Antimicrobial susceptibility of inducible Clindamycin resistant strains of Staphylococcus aureus (erythromycin resistant \& Clindamycin sensitive) were analysed by using Kirby Bauer's disc diffusion method as per CLSI guidelines. It was observed that penicillin, Ofloxacin, were $100 \%$ resistant, followed by Trimethoprim-sulfamethoxazole 108(95.5\%), Cefoxitin 101(89.4\%), Gentamycin 98(86.7\%) and 97(85.8\%) were resistant to Tetracycline \& Chloramphenicol while
Linezolid, Vancomycin, Ceftaroline, Rifampin were $100 \%$ sensitive.

\section{Conclusion}

Emergence of multidrug resistant strains of Staphylococcus species is an alarming and clinicians should aware about it. Use of Clindamycin without knowing the inducible resistance may lead to therapeutic failure. In present study, prevalence of inducible resistance was significant and we can conclude that the detection of inducible Clindamycin resistance on routine basis is mandatory for judicial use of the drug and proper institution of the therapy.

\section{References}

1. Jones RD, Kania SA, Rohrbach BW, Frank LA, Bemis DA. Prevalence of oxacillin-and multidrug-resistant staphylococci in clinical samples from dogs: 1,772 samples (2001-2005). J Am Veterinary Med Assoc 2007;230(2):221-7.

2. De Sousa MA, Sanches IS, Ferro ML, VazMJ, Saraiva Z, Tendeiro T, Serra J, De Lencastre H. Intercontinental Spread of a Multidrug-Resistant MethicillinResistant Staphylococcus aureusClone. J Clin Microbiol 1998;1;36(9):2590-6.

3. Leclercq R. Epidemiological and resistance issues in multidrug-resistant staphylococci and enterococci. Clin Microbiol Infection 2009;15(3):224-31.

4. Gordon RJ, Lowy FD. Pathogenesis of methicillinresistant Staphylococcus aureus infection. Clin Infectious Dis 2008;46(Supplement_5):S350-9.

5. Moreillon P, EntenzaJM, Francioli P, McDevitt D, Foster TJ, Francois P, Vaudaux P. Role of Staphylococcus aureus coagulase and clumping factor in pathogenesis of experimental endocarditis. Infection and Immunity. 1995;63(12):4738-43.

6. Enright MC, Robinson DA, Randle G, FeilEJ, Grundmann H, Spratt BG. The evolutionary history of methicillin-resistant Staphylococcus aureus (MRSA). Proceedings Natl Acad Sci 2002;99(11):7687-92.

7. Hiramatsu K, Cui L, Kuroda M, Ito T. The emergence and evolution of methicillin-resistant Staphylococcus aureus. Trends Microbiol 2001;9(10):486-93.

8. Rajaduraipandi K, Mani KR, Panneerselvam K, Mani M, Bhaskar M, Manikandan P. Prevalence and antimicrobial susceptibility pattern of methicillin resistant Staphylococcus aureus: A multicentre study. Ind J Med Microbiol 2006;24(1):34.

9. Roberts MC, Sutcliffe J, Courvalin P, Jensen LB, Rood J, Seppala H. Nomenclature for macrolide and macrolidelincosamide-streptogramin B resistance determinants. Antimicrobial agents chemotherapy 1999;43(12):282330.

10. Hatkar SS, Bansal VP, Mariya S, Ghogare HS. Antimicrobial Profile of Inducible Clindamycin Resistant Strains of Staphylococcus aureus Isolated From Clinical Samples. Int J Health Sci Res (IJHSR). 2014;4(6):99-103.

11. Prabhu K, Rao S, Rao V. Inducible clindamycin resistance in Staphylococcus aureus isolated from clinical samples. J Laboratory Physicians 2011;3(1):25.

12. Lewis JS, Jorgensen JH. Inducible clindamycin resistance in staphylococci: should clinicians and microbiologists be concerned? Clin Infectious Dis 2005;40(2):280-5.

13. SiberryGK, Tekle T, Carroll K, Dick J. Failure of clindamycin treatment of methicillin-resistant Staphylococcus aureus expressing inducible clindamycin 
resistance in vitro. Clin Infectious Dis 2003;37(9):125760.

14. Schreckenberger PC, Ilendo E, Ristow KL. Incidence of constitutive and inducible clindamycin resistance in Staphylococcus aureus and coagulase negative staphylococci in a community and a tertiary care hospital. J Clin Microbiol 2004;42(6):2777-9.

15. Ghogare HS, Hatkar SS, Bansal MP. Phenotypic detection of inducible clindamycin resistance among the clinical isolates of staphylococcus aureus by using D-test. IJHSR 2014;4(3):149-53.

16. AjanthaGS, Kulkarni RD, Shetty J, Shubhada C, Jain P. Phenotypic detection of inducible clindamycin resistance among Staphylococcus aureus isolates by using the lower limit of recommended inter-disk distance. Ind J Pathol Microbiol 2008;51(3):376.

17. Fiebelkorn KR, Crawford SA, McElmeel ML, Jorgensen $\mathrm{JH}$. Practical disk diffusion method for detection of inducible clindamycin resistance in Staphylococcus aureus and coagulase-negative staphylococci. J Clin Microbiol 2003;41(10):4740-4.

18. Manjunath V, Eshwar S, Ramya TG, Mridula RP, Sharma AD. test-its role in detection of inducible resistance to clindamycin in Staphylococcus aureus with special reference to MRSA. Int J Biol Med Res 2012;3(1):14302. 